Cinémas

Revue d'études cinématographiques

Journal of Film Studies

\title{
La voix over documentariste en fiction : entre factuel et fictionnel
}

\section{John Kristian Sanaker}

Volume 9, numéro 2-3, printemps 1999

Les Scénarios fictifs

URI : https://id.erudit.org/iderudit/024794ar

DOI : https://doi.org/10.7202/024794ar

Aller au sommaire du numéro

Éditeur(s)

Cinémas

ISSN

1181-6945 (imprimé)

1705-6500 (numérique)

Découvrir la revue

Citer cet article

Sanaker, J. K. (1999). La voix over documentariste en fiction : entre factuel et fictionnel. Cinémas, 9(2-3), 203-218. https://doi.org/10.7202/024794ar
Résumé de l'article

En se servant d'exemples québécois et français, l'auteur de cet article cherche à définir la fonction d'une certaine voix over, dite " documentarisante », dans des films de fiction. L'usage de cette voix over, propre au cinéma documentaire, signale l'existence d'une certaine réalité profilmique qui se greffe sur l'oeuvre de fiction; la disparition de cette voix nous dit que le film en question coupe ses liens manifestes avec la réalité profilmique pour établir son propre monde fictionnel. 


\title{
La voix over \\ documentariste en fiction: entre factuel et fictionnel
}

\section{John Kristian Sanaker}

\begin{abstract}
RÉSUMÉ
En se servant d'exemples québécois et français, l'auteur de cet article cherche à définir la fonction d'une certaine vioix over, dite "documentarisante", dans des films de fiction. Lusage de cette voix over, propre au cinéma dccumentaire, signale l'existence d'une certaine réalité profilmique qui se greffe sur l'œuvre de fiction; la disparition de cette voix nous dit que le film en question coupe ses liens manifestes avec la réalité profilmique pour établir son propre monde fictionnel.

\section{ABSTRACT}

Using examples from France and Quebec, the author of this article attempts to define the function of the socalled "documentarizing" voice-over in fiction films. This kind of voice-over, which is typical of documentary films, signals the existence of a certain pro-filmic reality that is grafted upon the works of fiction. Conversely, the disappearance of this type of voice-over informs us that the film is severing its ties with the profilmic reality to establish its own fictional world.
\end{abstract}

La voix over documentarisante en fiction : entre factuel et fictionnel

Avec Humain trop humain (1972), Louis Malle a fait une gageure: faire parler, en langage clair, des images de la réalité humaine sans aucun dialogue ni commentaire. Le film est tourné 
aux usines Citroën et nous fait surtout voir, et de façon minutieuse, ce qu'est le travail à la chaîne. Malle déclare lui-même avoir voulu "faire physiquement sentir ce que peut être la répétition des mêmes gestes pendant huit heures d'affilée" (cité par Claude Bouniq-Mercier dans Tulard, p. 1070). Il veut donc que les images seules disent la signification de ce que nous voyons, et nous pouvons supposer que l'intention du cinéaste a été de faire dire aux images que ce travail est inhumain, qu'il ne permet pas à l'ouvrier de s'épanouir en travaillant, que c'est un mode de travail qui prend le contrôle sur l'homme devenu machine.

Or, une telle lecture est loin d'être obligatoire. Rien ne m'empêche en principe d'y voir une démonstration de la maîtrise de l'homme dans le domaine de l'adaptation à ses conditions de travail, une faculté de manieur de machines et d'outils frôlant l'équilibrisme, donc une grande capacité de maîtriser cette situation supposée maîtrisante. Le fait que les acteurs eux-mêmes ne nous donnent pas de piste à suivre dans notre évaluation contribue à cette ambiguiité: il n'y a aucune réplique exprimant un mécontentement éventuel des ouvriers ni de gros plan montrant un visage en peine ou un regard désespéré qui auraient pu être notre ancrage pour une lecture univoque du visuel. Si on montrait le film à des adolescents prêts à choisir une formation professionnelle, serait-on certain que le film les dégoûterait de ce genre de travail?

Si on répond "oui" à la dernière question, il faudrait certainement ajouter des "parce que" révélant qu'un tel jugement serait nécessairement basé sur certaines idées préconçues en valeur dans une société donnée à une époque donnée. Et en tant que film postsoixante-huitard, Humain trop humain exploite probablement des topos comme l'idée d'aliénation du travail à la chaîne, ce qui a pu en faire un film relativement univoque en son temps. Mais cette signification est loin d'être donnée indépendamment de tout un contexte de valeurs, et si un certain nombre de spectateurs sont d'accord pour décoder le film de façon plus ou moins identique, c'est qu'ils adhèrent plus ou moins à ces mêmes valeurs.

Si on fait un effort pour porter un regard "objectif" sur le film et qu'on laisse tomber des critères d'évaluation qui sont 


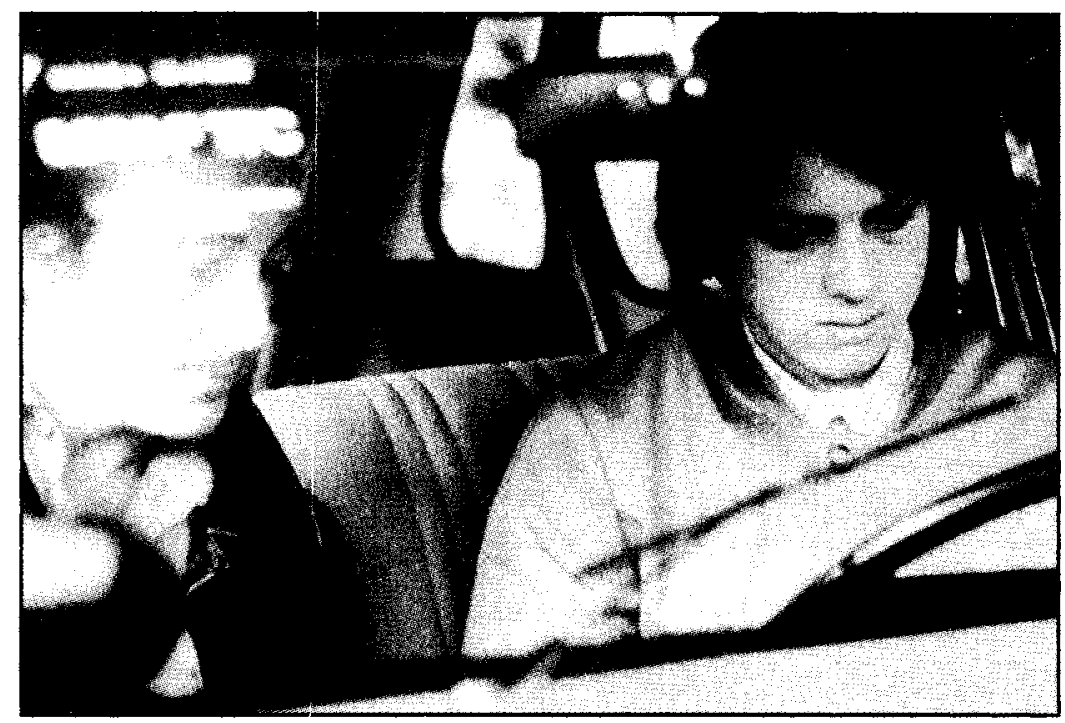

\section{Humain, trop humain de Louis Malle (1972)}

trop intimement liés à un contexte culturel particulier, on peut à la rigueur observer certaines scènes dont la teneur négative (travail à la chaîne incompatible avec le bonheur) semble avoir une valeur générale, et ceci grâce à certains phénomènes qui prennent une fonction de métaphores générales; c'est le cas, par exemple, pour les plans montrant l'avancée inexorable de la chaîne, et les pieds en gros plan qui reculent à l'approche de la mini-plateforme attachée à la chaîne, un ensemble figurant la machine à la chasse de l'homme, l'homme traqué par la machine. Mais c'est un fait que, faute d' "ancrage idéologique " ", nous nous trouvons devant une réalité filmique ouverte, où la lecture du spectateur individuel dépend largement des idées préconçues que ce spectateur a des choses montrées ${ }^{2}$.

Prenons maintenant un exemple à l'autre extrémité de l'éventail des possibilités: un film à ancrage maximal avec un texte lu qui veut transformer une réalité visuelle peu marquée en un message univoque. Le Temps des bouffons (1993) est un filmpamphlet du Québécois Pierre Falardeau; il s'agit d'un courtmétrage à commentaire ininterrompu, comportant deux parties bien distinctes: la première partie est composée d'extraits des 
Mâtrtres fous (1956) de Jean Rouch (les commentaires de Falardeau remplaçant ceux de Rouch), la seconde est un reportage sur la fête annuelle (et en même temps le $200^{\circ}$ anniversaire) du Beaver Club de Montréal, un club social.

Les images empruntées aux Maîtres fous sont propres à confirmer le test célèbre de Chris Marker dans Lettre de Sibérie (1958). Falardeau enlève donc le commentaire de Rouch, commentaire d'ethnologue qui vise la connaissance des phénomènes exotiques observés - leur signification apparente aussi bien que leur présupposés —, pour y proposer le sien, commentaire à valeur politique, idéologique. Pour Rouch, il s'agit de phénomènes à expliquer tels qu'ils sont vécus par les acteurs euxmêmes; pour Falardeau, il s'agit de modifier la signification des images de Rouch en situant les phénomènes dans un contexte plus large de politique et d'histoire coloniales, d'opposition entre colonisés et colonisateurs.

Cette perspective est ensuite poursuivie dans le commentaire sur les images produites par Falardeau lui-même. On y voit la très haute société québécoise en tenue de soirée; les gens se divertissent gentiment en mangeant, en buvant, en chantant et en dansant. L'ambiance est excellente et tout ce monde riant est habillé avec élégance, soit en tenue de soirée, soit en costumes anciens, avec fausse barbe, etc., pour honorer leurs ancêtres fondateurs du club: les premiers grands commerçants de la fourrure.

Le travail de la caméra est plutôt neutre. Si l'on visionne le film sans commentaire, les images nous paraissent ouvertes, pour ne pas dire plates, et une prise de position éventuelle serait probablement déterminée par ce que nous pensons en général de gens du beau monde qui se divertissent ainsi (ridicules, amusants, brillants, piteux, etc. ${ }^{3}$ ).

Contre ces images assez quelconques, Falardeau projette un texte d'une grande virulence. D'une part, il nomme les acteurs qu'on voit en déchirant leur apparence bonhomme pour montrer ce qu'il considère leur vrai visage d'exploiteurs pervers:

Toute la rapace est là : des boss pis des femmes de boss, des barons de la finance, des rois du pizza congelé, des mafiosi de l'immobilier. Toute la gang des bienfaiteurs 
de l'humanité, des charognes à qui on élève des monumerts, des profiteurs qui passent pour des philanthropes $[\ldots]^{4}$.

D'autre part, la célébration du bicentenaire du club et les costumes historiques de ceux qui se trouvent à la table du président lui permettent de donner sa version personnelle de l'histoire du Québec, une longue histoire d'exploitation où la "mafia» anglaise prend le pouvoir pour tout acheter: "Les terres, les honneurs, les médailles, le pouvoir, tout ce qui s'achète. La gang de la fourrure forme lentement l'élite de la société. Les voleurs deviennent tranquillement d'honorables citoyens. "Et de toute cette "marde" que Falardeau a devant les yeux, les plus détestables sont les "collabos bilingues", l'élite francophone qui accepte de jouer le jeu de l'ennemi en acceptant de parler leur langue 5 .

Falardeau y va donc avec toute sa haine de gauchisteindépendantiste pour nous dire que la réalité n'est pas ce que nous voyons (ni ce que nous dit Jean Rouch). Sous l'apparence innocente de la fêtte du Beaver Club, il voit, et essaie de nous faire voir, une réalité qui est cachée, mais dont les symptômes sont visibles.

Ce «forçage» (Daney, p. 24 $4^{6}$ ) de l'image n'est nullement le produit d'un commentaire neutre dont il est vain de chercher les présupposés énonciateurs ${ }^{7}$. Par contre, Falardeau s'y investit par le timbre et l'expressivité de sa voix (bien connue au Québec) qui nous disent très clairement sa haine pour ce monde d'exploiteurs pourris et son mépris profond pour tous ces acteurs historiques qui n'ont pas mieux contribué à écrire l'histoire du Canada français et du Québec. Contrairement à la voix neutre du documentaire traditionnel, celle de Falardeau est donc hautement personnalisée et ne cache en rien le fait qu'elle véhicule une prise de position individuelle. Le Temps des bouffons est un produit bimodal où le texte lu essaie, à travers l'investissement affectif et idéologique de son producteur, de déterminer de façon très précise la signification du visuel plutôt flou et insignifiant ${ }^{8}$. 


\section{Une réalité à construire}

Ce qui m'intéresse, ici, n'est pas d'abord l'opposition idéologique et méthodologique entre documentaire classique à commentaire et cinéma direct; dans une telle optique dichotomique, l'attitude didactique, voire autoritaire, qu'implique la pratique même du commentaire s'opposerait à l'attitude ouverte du direct qui permettrait à la réalité même de prendre forme sans passer par le travail médiateur du commentaire.

Je m’intéresse plutôt à l'idée selon laquelle le documentaire, genre qui par définition établit des rapports plus ou moins intimes avec la réalité, aurait à s'efforcer pour devenir une construction pleine, alors que le film de fiction constitue un monde autosuffisant et fermé. Le film de fiction construit son propre univers complet, sans présupposer l'existence d'une réalité non fictionnelle où il s'inscrit, tandis que le documentaire reste, par définition, ouverte sur le monde à propos duquel il prétend nous "documenter", mais sans jamais pouvoir tout montrer, compte tenu que chaque "document» est nécessairement incomplet ${ }^{9}$.

C'est dans cette optique que j'ai présenté mes deux exemples préliminaires. Humain trop humain est un défi au documentaire classique aussi bien qu'au cinéma direct, dans la mesure où il prétend pouvoir montrer de façon suffisamment claire la teneur de la réalité qui est l'objet du film, sans le commentaire explicatif de celui qui porte son regard sur cette réalité, ni un dialogue donnant la parole aux acteurs muets qu'on observe. Le Temps des bouffons, par contre, nous met sans intermittence en présence d'un commentaire qui, non seulement dit la réalité que nous voyons en lui donnant une valeur que nous sommes peu disposés à y voir sans ce commentaire, mais qui aussi va loin audelà du visible pour nous donner une vision de l'histoire où les personnages présents sont des acteurs assez secondaires et occasionnels.

Tendanciellement, le documentaire serait donc, vu son ouverture sur la réalité où il s'inscrit, un genre dont le niveau «diégétique" (images, répliques, son réel) est à compléter par un commentaire assurant cette inscription; il serait marqué par un manque, un vide à remplir là où le film de fiction standard 
constitue par définition son propre monde complet (différence tendancielle attestée par la très haute fréquence de documentaires à voix over, la plus faible fréquence de films de fiction à voix over). Un exemple éloquent pour illustrer cette différence de base serait tous les documentaires sur la Deuxième Guerre mondiale essayant de reconstruire ce passé à travers des traces à expliquer, à compléter par opposition à des films de fiction inventant ce même passé comme quelque chose de complet; du côté documentaire, il y a, par exemple, Nuit et brouillard (Resnais, 1956), Shoah (Lanzmann, 1974-1985), Hôtel Terminus (Ophuls, 1988), où un commentaire nous aide à reconstruire les atrocités de la guerre à partir des indices d'aujourd'hui: un paysage devient un camp de concentration chez Resnais, un tas de pierres devient un four crématoire chez Lanzmann, l'intérieur d'une prison nous mène à la réalité de la torture chez Ophuls; du côté fiction, il y a Le Choix de Sophie (Sophie's Choice, Pakula, 1982) et La Liste de Schindler (Schindler's List, Spielberg, 1993) qui mettent en scène la catastrophe comme une réalité pleine qui s'explique par l'ensemble des éléments diégétiques concourant pour créer la cohérence propre au film en question.

\section{Un régime mixte : le commentaire documentarisant en fiction}

Un certain type de films à régime rhétorique mixte est particulièrement intéressant dans cette optique: celui où un commentaire de type documentarisant cède la place à une fiction autosuffisante qui rend le commentaire superflu, voire incompatible avec les composantes filmiques qui contribuent à établir sa plénitude, sa clôture en tant que fiction. Dans ce genre de film, on peut donc observer la volonté de donner au film une valeur de document, tout en lui assurant le statut de film de fiction; et pour établir ce contrat de lecture double, la présence/absence d'une voix over y est pour beaucoup ${ }^{10}$.

Commençons par un exemple curieux et radical du cinéma québécois. On est loin du soleil (1970) est à l'origine un film de commande; l'Office national du film a demandé à Jacques Leduc de faire un film sur une personnalité célèbre au Québec: le frère André, thaumaturge. Le film commence par 2 min $50 \mathrm{sec}$ d'écran noir accompagné par un commentaire qui nous 
résume la vie du frère André: naissance, enfance, jeunesse, départ en Nouvelle-Angleterre, retour, prononciation des vœux, carrière de guérisseur, mort. À la fin apparaît pour douze secondes son portrait photographique en vieil homme.

Ensuite, une entraînante musique de rock accompagne pendant $3 \mathrm{~min} 10 \mathrm{sec}$ un paysage urbain où l'on observe la vie au quotidien; il y a alternance d'images qui relèvent visiblement du tournant du siècle (donc de l'époque du frère André) et d'images contemporaines provenant du tournage du film. La musique prend fin au moment même où nous voyons une femme sortir de chez elle pour aller en ville; en même temps apparaît le son réel, et comme la dame n'aperçoit pas la caméra même si l'équipe de tournage doit nécessairement encombrer sa petite véranda qu'elle traverse pour descendre dans la rue, nous avons le droit d'en conclure que nous sommes en régime de fiction.

En effet, la séquence "paysage urbain» a fonctionné comme un lien entre le prologue documentaire avec écran noir et le film proprement dit, un film de fiction sur divers membres d'une famille québécoise de l'époque du tournage du film, des personnages censés représenter des défauts et des qualités propres à ce peuple québécois qu'a servi le frère André (patience, résignation, soumission). Le film de Leduc constitue donc un détournement de la commande faite par l'ONF, dans la mesure où il refuse de façon démonstrative d'être un documentaire sur le frère André (l'écran noir), tout en proposant des documents (le texte lu en voix over et la photo) dont la fonction serait de sous-tendre le film qu'on va voir, film dont la fiction serait propre à nous donner, par voie analogique, une matière à réflexion suscitant une attitude critique envers une société où un faiseur de miracles peut jouir d'un tel prestige.

Dans ce film, la coupure entre les deux régimes est donc très nette. Le plus souvent, la délimitation se fait de façon plus discrète, plus souple. Regardons maintenant comment la délimitation s'opère dans un exemple célèbre du cinéma français: La Bataille du rail (Clément, 1946). Après des renseignements sur la situation de la France coupée en deux, ce film se présente comme une reconstitution de l' "armée" des chemins de fer 
français et de sa contribution à la «désorganisation des transports, à la défaite allemande dans la bataille de la libération" (texte sur l'écran après les génériques). Ensuite, quelques scènes qui marquent le début de l'action proprement dite annoncent de façon univoque qu'il s'agit d'un film de fiction autosuffisant: contrôle des pièces d'identité dans un train en partance, cheminot qui interdit à un Allemand de monter dans sa locomotive (où il a caché des partisans), tracts cachés en dessous d'un wagon, fugitif caché dans une caisse à chien, etc.

Cependant, ces quelques scènes sous forme de mosaïque ne font que créer une atmosphère: celle de la Résistance. Elles ne nous renseignent pas sur ce qui sera la trame du film: le dérèglement du transport allemand opéré par les cheminots français. Et voilà que, après $7 \mathrm{~min} 30 \mathrm{sec}$, s'introduit une voix over explicative: "Et maintenant, voilà comment cela se passait: (on voit un personnage entrer au "Poste de commandement") de ce poste de commandement $[. .$.$] , etc." On observe des fonctionnaires exer-$ çant diverses fonctions, et un officier allemand qui s'introduit: "Ce monsieur galonné réclame [...], etc.» Les fonctionnaires refusent visiblement de collaborer, et le commentaire nous explique l'importance du réseau de communication: "N'oubliez pas: le poste de commandement est en relation directe avec toutes les gares de la région. " Et après des renseignements détaillés sur le double jeu des opérateurs, leur permettant d'introduire «des cailloux qui font grincer la machinerie» allemande, le commentateur se retire ( $M o i$, je n'ai plus rien à vous expliquer»), estimant qu'il nous en a assez dit pour que nous sachions déchiffrer nous-mêmes la vraie signification des actes principaux du film de fiction que sera désormais La Bataille du rail.

Il s'agit donc d'un film de fiction reconstruisant de si près des événements réels que le besoin est senti de nous fournir des clés pour bien saisir le côté technique du film, tous ces détails qui constituent des composantes importantes de l'action, mais qui ne sont pas propres à intéresser le volant fiction, qui mettra plutôt en évidence la psychologie des acteurs (courage, hésitations, inventivité des cheminots) et le côté spectaculaire du film en tant que film d'action (surtout le déraillement du train allemand"). 


\section{Le Dernier Métro}

La façon exemplaire dont Truffaut se sert de ce régime mixte dans Le Dernier Métro (1983) mérite qu'on s'y arrête pour un moment. Comme nous le savons, la guerre et l'Occupation constituent un arrière-plan dont l'importance est primordiale pour le déroulement de l'action; le comportement de chacun des personnages est étroitement déterminé par ce contexte contraignant.

Cependant, le monde fictionnel que crée le film est remarquablement limité: une visite de Marion à la Propagandastaffel, une sortie au restaurant, une scène dans les locaux du journal de Daxiat et dans une église, ce qui rompt avec l'impression générale de huis clos. On reste enfermé dans le petit monde du théâtre Montmartre avec son bureau, ses couloirs, sa salle, sa cave et sa rue adjacente (à peine perçoit-on la chambre d'hôtel de Marion comme appartenant à "l'extérieur").

Ce monde clos est donc encadré par le monde réel de la France pendant l'Occupation et est en même temps soumis aux lois et à l'atmosphère qui y règnent. Cette atmosphère est suggérée dès la première séquence du film qui nous fait entendre une voix over documentarisante:

Paris, septembre 1942. Depuis deux ans, l'armée allemande occupe la moitié nord de la France. La séparation entre la zone occupée et la zone libre constitue une sorte de frontière qui traverse horizontalement le pays. En zone occupée, le couvre-feu vide les rues après 11 heures du soir et, pour les Parisiens, il est terriblement important de ne pas rater le dernier métro, etc.

Ces renseignements, qui nous font penser au texte préliminaire de La Bataille du rail, sont accompagnés d'images hétéroclites ayant toutes une fonction illustrative: scènes de rue, carte figurant la France coupée en deux, photos en couleur ou en noir et blanc évoquant la vie du Paris occupé.

Vers la fin de ce prologue, le commentaire nous apprend que les théâtres sont des lieux où se pressent les Parisiens «qui ont froid chez eux". Ainsi on nous prépare à abandonner le cadre général à valeur documentaire pour nous approcher du monde fictionnel où va se passer l'action. Le passage de l'un à l'autre se 
fait adroitement par le biais de la voix over: "Au théâtre Montmartre, une pièce est en répétition et pourtant le directeur, Lucas Steiner, a quitté la France précipitamment. Il n'avait pas le choix. " Ce texte est lu contre une image du théâtre Montmartre sur laquelle se "superimpose" le portrait de Steiner, deux composantes essentielles de la fiction à venir, qui sont d'abord documentarisées par leur introduction dans le cadre historique.

Cependant, le déroulement de la partie fiction du film nous apprend que Steiner n'est pas parti, mais qu'il se cache dans la cave du théâtre. Le commentaire est donc implicitement décrit comme une voix "officielle", extérieure, une voix qui perd sa fonction et son pouvoir dès que le prologue "la France sous l'Occupation" cède la place à la fiction "Marion et sa lutte pour faire marcher son théâtre ", pièce de fiction de type autosuffisant dont le début est marqué par un écran noir auquel succède la scène de rue où Bernard drague Arlette avec la première occurrence de son réel.

A sept minutes de la fin du film, le commentaire fait sa réapparition (pour une durée de trois minutes) et opère en même temps une réouverture du monde fictionnel sur le monde extérieur. Et du même coup, les personnages cessent d'être confinés à l'histoire de Marion et de son théâtre, pour entrer dans d'autres rôles dans la partie du film que nous avons appelée "la France sous l'Occupation". À tour de rôle Nadine, Arlette et Jean-Loup sont présentés par la voix over avec images illustratives dès qu'ils sortent du monde clos de la fiction, pour regagner le monde extérieur de la fin de l'Occupation. Le cas de Daxiat est peut-être le plus intéressant. Au long de la fiction, ce journaliste du journal collaborateur Je suis partout est entré à plusieurs reprises dans le monde de la fiction pour y jouer un rôle étroitement limité à sa fonction comme trouble-fête dans l'optique de Marion et de ses collaborateurs. Vers la fin du film (et de l'Occupation), il devient du coup un personnage officiel qui tombe dans le domaine de la voix over documentarisante, le salaud qui a du mal à sauver sa peau à la Libération :

Daxiat ne devra son salut qu'à la fuite. Le journaliste de Je suis partout est devenu un homme de nulle part. Il a perdu un oil dans le terrible bombardement de 
Hambourg. Il a suivi un moment le maréchal Pétain à Sigmaringen. Puis, de monastère en couvent, il réussira à gagner l'Espagne. Condamné à mort par contumace, il mourra d'un cancer à la gorge dans les années soixante.

Les images illustratives montrent un Daxiat en uniforme et en fuite qui, un bandeau sur l'œil, traverse des ruines en train de brûler (donc des images correspondant à l'information sur le bombardement de Hambourg). Daxiat et les autres sortent donc de la fiction; ils cessent d'être "personnages" pour devenir "citoyens ${ }^{12}$; ; et les régimes avec/sans voix over correspondent intimement aux deux modes de ce film à cheval entre les genres ${ }^{13}$.

\section{Travelling avant}

Un autre film qui inscrit la matière fictionnelle dans un cadre historique très précis est Travelling avant (Tacchella, 1987). Images aussi bien que dialogues et commentaires sont truffés de références à cette époque et ce lieu que célèbre Tacchella: les débuts de la vraie cinéphilie à Paris à la fin des années quarante. Et la partie documentaire de ce film "mixte" occupe une place beaucoup plus importante que dans Le Dernier Métro. En effet, si on essaie d'identifier ce qui est trame purement fictionnelle, on retient une matière bien maigre. Il est toujours possible d'isoler du reste du film une intrigue amoureuse avec de jeunes couples qui se forment et se désintègrent, mais c'est évident que personne n'irait voir le film pour ces querelles amoureuses et ces scènes intimes.

Ce n'est pas la passion amoureuse des jeunes qui donnent au film son poids, c'est leur passion "cinéphilique». À tout moment, dans toute situation, ils sont prêts à citer des films, à se référer à tel acteur, à tel metteur en scène, à citer telle réplique célèbre, et même à transformer tel vécu en matière filmique en discutant éclairage et plans; la somme de toutes ces références constitue une véritable histoire de la culture "cinéphilique» parisienne avant la Nouvelle Vague.

Comme cette masse référentielle est répandue un peu partout dans le film, la fonction de la voix over n'est plus, comme dans 
Le Dernier Métro, de délimiter clairement ce qui est valeur de document et ce qui ne l'est pas. Il s'agit plutôt d'une augmentation du poids du référentiel, et les six occurrences ont rapport exclusivement au côté "cinéphilique" de l'histoire (les habitudes des cinéphiles, le phénomène des ciné-clubs, l'existence de la Cinémathèque, d'Objectif 49).

Cependant, la sixième et dernière occurrence, qui clôt le film, prend une fonction documentarisante toute particulière, et on a rarement vu d'opération de mixtion des genres plus radicale, efficace et momentanée. Les membres du noyau de la bande, Donald, Nino et Barbara, ont décidé de partir ensemble pour assister au "Festival du film maudit " à Biarritz. Sur l'image de la voiture qui démarre, nous entendons le commentaire suivant:

Le Festival du film maudit se déroula à Biarritz du 29 juillet au 5 août 1949 sous la présidence de Jean Cocteau. Ce fut le premier festival uniquement consacré aux cinéastes et à leurs œuvres. On logea dans un lycée les jeunes cinéphiles qui avaient décidé de suivre le Festival. L'un d'eux s'appela François Truffaut.

On voit bien comment cette dernière voix over documentarisante diffère des autres moyens utilisés dans ce film pour renforcer son caractère de document sur l'époque. Au cours de l'action, image et dialogue font entrer dans la fiction Julien Duvivier et L'Atalante, Lauren Bacall et Le Silence de la mer, Objectif 49 et Orson Welles; mais la valeur de ces éléments référentiels est toujours liée à l'expérience personnelle des jeunes personnages. Tacchella fait entrer le réel dans son film en le fictionnalisant. La dernière voix over, par contre, voix détachée d'un historien, nous fait sortir du docu-fictionnel pour entrer dans la réalité historique où un certain François Truffaut, qui n'a pas été objet de la même fictionnalisation que par exemple Duvivier, participe à un Festival de cinéma bien réel. La voix over documentarisante sert donc non plus à évaluer la valeur documentaire du réel fictionnalisé, mais à situer la fiction que nous venons de voir par rapport à cette réalité historique qui n’a cessé de la nourrir. C'est comme si Tacchella, en minimisant l'importance de la fiction proprement dite et en obligeant ses 
jeunes personnages à subordonner leur vie privée et sentimentale à leur fonction comme véhicule d'un document sur l'époque, a voulu conclure en disant que les jeunes cinéphiles ont bien mérité, en compensation pour leur manque de liberté individuelle, de côtoyer Truffaut pendant le Festival et ainsi, d'entrer de façon proleptique dans l'histoire du cinéma français!

Les relations entre fiction et réalité au cinéma sont complexes. Par ces quelques exemples, j'ai voulu montrer comment un certain usage de la voix over documentarisante peut servir à délimiter ce qui est base factuelle et ce qui est construction fictionnelle, tout en assurant l'inscription efficace du fictionnel dans un cadre factuel.

En même temps, les exemples étudiés démontrent une des différences principales entre documentaire et film de fiction (si l'on croit encore à la possibilité d'établir une telle différence!) : la voix over de type documentarisante illustre l'incomplétude nécessaire de tout film documentaire par rapport au monde réel documenté, alors que la disparition ou l'absence du commentaire dans les films analysés nous font percevoir le règne de l'image-dialogue comme un signe sûr de clôture et de complétude fictionnelles.

\section{Université de Bergen}

\section{NOTES}

1 Voir Roland Barthes sur "l'ancrage idéologique» et la fonction du texte qui «dirige le lecteur entre les signifiés de l'image" (L'Obvie et l'obtus, Paris: Seuil, 1982, p. 32).

2 Je cite aussi D'Est de Chantal Akerman (1993), film sans dialogue ni commentaires où des images de l'ancienne Europe de l'Est peuvent probablement évoquer chez le spectateur pitié, douceur, tristesse, nostalgie, etc., la réaction dépendant de ce qu'on penserait de la lenteur de la vie représentée et de l'absence éventuelle de choses qui sont souvent valorisées, mais qui sont loin d'avoir une valeur absolue (voitures, vitesse et efficacité, activité énergétique).

3 Les images ont été tournées en 1985 et, vu leur caractère ouvert, on n’est pas étonné d'apprendre qu'à l'époque, Falardeau ne savait pas encore exactement à quelle fin utiliser ce matériau.

4 Le texte est paru dans Pierre Falardeau, La liberté n'est pas une marque de yogourt (Montréal: Stanké, 1995).

5 Comme Roger Landry, le président du club, qui fait son discours alternativement en français et en anglais et qui est donc, au niveau du reportage, une illustration du postulat de Falardeau. 
6 I.e terme est emprunté à Serge Daney, qui l'utilise pour désigner la voix «qui est roujours parallèle au défilé des images", et qui, "surimposée après coup à l'image" s'adresse "au spectateur avec lequel elle fait alliance, contrar, stur le dos de l'image" ("L'orgue et l'aspirateur $*$, Cahiers du cinéma, n" 278-279, 1977, p. 24).

7 Voir Pascal Bonitzer (Le Regard et la voix, Paris: Union générale d'éditions, 1976, p. 30-41) pour qui "[...] dans l'espace hétérogène, divisé du documentaire, la voix off interdit les questions de son énonciateur, de son lieu et de son temps" (p. 33). L'exception est le cas, pour le cinéaste très risqué, où se perçoit dans la voix over «la passion partisane" (p. 39); Bonitzer renvoie à la démonstration de Marker dans Lettre de Sibérie, qui nous montre surtout que "[...] le commentaire ne doit pas faire violence à l'image " (p. 38).

8 Le procédé de Falardeau me semble correspondre à ce que Bonizzer définit comme du cinéma militant, qu'il oppose au documentaire classique: "Il faut qu'il soit tout à fait autre chose. [...] ce qui se donne au titre de cinéma militant ne commence jamais que là où finit le documentaire classique, dans ce que celui-ci étouffe er gomme: le sujet parlant" (Le Regard et la voix, Paris: Union générale d'éditions, 1976, p. 46).

9 Pour une bonne introduction à ces problèmes, voir André Gardies, Le Récit filmique (Paris: Hachette, 1993), chapitres 3 et 8.

10 D’après Pascal Bonitzer (Le Regard et la voix, Paris: Union générale d'éditions, 1976 , p. 32), cette "division du champ filmique" opérée par l'introduction d'une voix over "sans communication convenue avec [l'espace] qu'ouvre l'image" est si rare en fiction qu'elle produit nécessairement un "effer d'érrangeté". Les exemples que j'ai trouvés montrent plutôt qu'il s'agit d'un phénomène suffisamment courant pour constituer une sous-catcigorie narrative qu'il vaut la peine d'étudier, dans des films oì l'on est frappé par la régularité du phénomène et non pas par son étrangeté.

11 Un tel résidu factuel peut, en principe, lier un film de fiction à n'importe quel sous-genre documentaire. Le double régime s'observe par exemple dans des films aussi différents que S'en fout la mort (Denis, 1990), où la voix over nous introduit au monde des combats de cogs, et Orphée (Cocteau, 1950), oì la voix de l'auteur nous donne un bref cours de mythologie grecque en nous disant ce quil faut savoir sur le mythe d'Orphée pour pouvoir suivre l'acrion de son film. Qu'on se souvienne aussi de Duvivier qui dans Pépé le Moko (1937), en pleine fiction, introduit un morceau de film d'ethnologue à la Jean Rouch : la présentation que fait l'inspecteur de police de la Casbah, avec voix over et images illustratives, présentation destinée à informer le collègue venu de Paris.

12 C'est le même effet qui se produit à la fin d'Aut revoir les enfants (Malle, 1987), ce film dont les événements principaux ont été "vécus" par le cinéaste lui-même (interview, Séquences, n'133, mars 1988, p. 22). Malle n'a même pas changé le nom du garçon juif, et la voix over terminale a clairement un effet documentarisant en disant le destin du "citoyen" Jean Bonnet, élève dans la classe du jeune Louis Malle: "Bonnet, Négus et Dupré sont morts à Auschwitz, le père Jean à Mathausen. [...] Plus de 40 ans ont passé, er jusqu'a ma mort, je me rappellerai chaque seconde de ce matin de janvier."

13 On retrouve la même voix over documentarisante dans un autre film de 'Truffaut: L'Histoire d'Adele $H$ (1975). Au début du film, le commentaire sert à situer l'action dans l'histoire; vers la fin, laction à laquelle nous venons d'assister est situéc par rapport à la vie politique en France et à la vie de Victor Hugo.

Nous pouvons observer un autre exemple intéressant dans Matelot 512 (René Allio, 1984); le commentaire très fréquent accompagne régulièrement la carrière de Max en insistant surtout sur les aspects publics de son existence: voyages, vie de

I a voix over documentariste en fiction: entre factuel et fictionnel 
soldat, etc. Or, parfois la voix over se tait pour des séquences entières montrant le côré privé de sa vie. À cet égard, la première occurrence sans commentaire est caractéristique: sa nuit d'amour secrète avec sa future femme, un morceau de pure fiction romanesque isolé du contexte "carrière" auquel il est littéralement volé.

\section{OUVRAGES CITÉS}

Barthes, Roland. "Rhétorique de l'image", in L'Obvie et l'abtus. Paris: Seuil, 1982.

Bonitzer, Pascal. Le Regard et la voix. Paris: Union générale d'éditions, 1976.

Daney, Serge. "Lorgue et l'aspirateur », Cahiers du cinéma, $\mathrm{n}^{\text {"s }}$ 278-279 (1977), p. 19 27.

Falardeau, Pierre. La liberté n'est pas une marque de yogourt. Montréal: Stanké, 1995. Gardies, André. Le Récit filmique. Paris: Hachette, 1993.

Tulard, Jean. Guide des films. Paris: Laffont, 1990. 